\title{
Nelly Labère, Défricher le jeune plant. Étude du genre de la nouvelle au Moyen Âge
}

\section{Maria Colombo Timelli}

\section{(2) OpenEdition}

\section{Journals}

\section{Édition électronique}

URL : http://journals.openedition.org/studifrancesi/9789

DOI : ERREUR PDO dans /localdata/www-bin/Core/Core/Db/Db.class.php L.34 : SQLSTATE[HY000]

[2006] MySQL server has gone away

ISSN : 2427-5856

\section{Éditeur}

Rosenberg \& Sellier

Édition imprimée

Date de publication : 1 octobre 2007

Pagination : 418-419

ISSN : 0039-2944

\section{Référence électronique}

Maria Colombo Timelli, « Nelly Labère, Défricher le jeune plant. Étude du genre de la nouvelle au Moyen Âge », Studi Francesi [En ligne], 152 (LI | II) | 2007, mis en ligne le 30 novembre 2015, consulté le 08 janvier 2021. URL : http://journals.openedition.org/studifrancesi/9789; DOI : https://doi.org/10.4000/ studifrancesi.9789

Ce document a été généré automatiquement le 8 janvier 2021.

\section{(c) $($ ) $\odot \odot$}

Studi Francesi è distribuita con Licenza Creative Commons Attribuzione - Non commerciale - Non opere derivate 4.0 Internazionale. 


\title{
Nelly Labère, Défricher le jeune plant. Étude du genre de la nouvelle au Moyen Âge
}

\author{
Maria Colombo Timelli
}

\section{RÉFÉRENCE}

NELLY LABÈRE, Défricher le jeune plant. Étude du genre de la nouvelle au Moyen Âge, Paris, Honoré Champion, 2006 («Bibliothèque du Xve siècle», LXIX), pp. 1061.

1 Depuis plus d'un siècle maintenant, les critiques s'interrogent sur la naissance de la nouvelle en France: pour retrouver les prodromes de ce débat on n'a qu'a se reporter au long compte rendu que Gaston Paris consacra dans le "Journal des Savants" de mai et juin 1895 au livre de Pietro Toldo, Contributo allo studio della novella francese del XV e XVI secolo, considerata specialmente nelle sue attinenze con la letteratura italiana (Roma, 1895). Si les accents de la polémique sur l'origine italienne du genre se sont estompés depuis lors, le problème de la génèse du genre est loin d'être résolu, et les études se succèdent portant tant sur des questions purement littéraires que sur les aspects lexicaux impliqués dans toute interrogation sur la naissance d'une nouvelle désignations littéraire (voir par exemple l'article stimulant de Roger Dubuis, Le mot 'nouvelle' au Moyen Age: de la nébuleuse au terme générique, dans La Nouvelle. Définitions, transformations, Presses Universitaires de Lille, 1990, pp. 13-27).

2 Nelly Labère vient enrichir le dossier avec une étude fondée sur un corpus de recueils qui couvre tout le $\mathrm{xv}^{\mathrm{e}}$ siècle: Quinte Joyes de Mariage, traduction du Decameron par Laurent de Premierfait, Nouvelles dites de Sens, Arrêts d'Amour de Martial d'Auvergne, Cent Nouvelles nouvelles, Evangiles des Quenouilles, traduction du Liber facetiarum de Poggio Bracciolini par Guillaume Tardif. Il s'agit d'un volume imposant, organisé en quatre parties; le lecteur spécialiste y trouvera des réflexions intelligentes appuyées sur une 
bibliographie richissime, alors que les amateurs pourront profiter des lectures croisées et des analyses, par endroits très fines, des ouvrages examinés.

Une telle étude ne pourrait négliger la question terminologique; la première partie (Les emplois de la nouvelle, pp. 43-160) essaie donc de faire le point sur les emplois non génériques du mot, puis de figer son passage à l'acception littéraire. La question est de poids, d'une part parce que le substantif 'nouvelle' remonte au xi ${ }^{e}$ siècle et que, par conséquent, au $\mathrm{xv}^{\mathrm{e}}$ il ne constitue, au mieux, qu'un néologisme de sens; d'autre part parce que l'influence du mot italien 'novella' (par le biais de la traduction de Laurent de Premierfait, qui remonte à 1414) est indéniable. Qui plus est, le transfert du 'jeune plant' - selon l'efficace métaphore de Nelly Labère - sur le sol français se fait dans un terrain déjà riche en genres 'brefs': la deuxième partie (Un genre protéiforme, pp. 161-287) se veut par conséquent une mise au point sur la polygénèse de la nouvelle et sur les rapports privilégiés qu'elle entretient avec le fabliau; une réflexion à part est consacrée aux aspects qui concrétisent la dimension ludique (rire, passe-temps, jeu, nouveauté) et à la prétendue véracité des récits.

4 Le rapport avec la 'novellistica' italienne fait l'objet de la troisième partie, centrée tout spécialement sur le Decameron et sur le Liber facetiarum en rapport avec leurs traductions respectives. La réception française des deux ouvrages est située d'abord dans un vaste panoramique qui encadre le succès et la tradition, manuscrite et imprimée, des deux recueils. Nelly Labère passe ensuite à une analyse serrée des pratiques traductives pour aboutir au rôle joué par les deux traducteurs français, Laurent de Premierfait et Guillaume Tardif, qui, chacun à sa manière, se font auteurs bien plus que simples médiateurs linguistiques.

5 La dernière partie accueille les suggestions avancées depuis maintenant une vingtaine d'années par la critique, en mettant l'accent sur la modalité de transmission des nouvelles au sein de ce qui paraît contribuer strictement à leur définition même: le recueil (Recueil et nouvelles, pp. 459-677). Bien entendu, toute généralisation serait impossible, et ce sont les recueils mêmes objets de son étude qui déterminent les principes organisateurs explicités par Nelly Labère: organisation par emprunts, matière, fiction, série.

6 Menant moi-même une étude sur le recueil de nouvelles sans aucun doute le moins étudié du $\mathrm{xv}^{\mathrm{e}}$ siècle (les Nouvelles de Sens, partiellement éditées par Ernest Langlois en 1908, puis quasi ignorées par la critique), j'ai été particulièrement intéressée par les passages où Nelly Labère examine ce texte. Si j'ai admiré la finesse de certaines de ses propositions, par exemple à propos de l'organisation interne du recueil (pp. 555-556), je n'ai pas pu éviter de remarquer des imprécisions (entre autres, la citation du texte aux pp. 236-237 fourmille de fautes, et une ligne a même sauté en bas de la p. 236: «Sire, c'est pour la fille d'ung roy de grande noblesse, qui me l'a baillee a garder par telle condicion que, se ne la luy rens saine et nette comme il la m'a baillee, qu'il me fera mourir...", bel exemple de saut du même au même de la part d'un copiste moderne...) et quelques excès, dus sans doute à l'enthousiasme: ainsi lorsque Nelly Labère affirme que l'emploi du mot 'nouvelle' dans l'intitulé du premier texte du recueil, et le recours au mot 'nouvelletez' dans le chapitre XXXV marqueraient «le passage d'un emploi générique à un emploi moral»; si Langlois déjà, et avant lui Vossler, avaient remarqué le parcours ascensionnel qui caractérise les récits au recueil de Sens, il me semble que l'on va ici un peu trop loin dans l'attribution d'une volonté si subtile à un compilateur qui ne brille ni par sensibilité littéraire et linguistique ni par esprit de système. 
7 Le gros livre de Nelly Labère comprend encore une série importante de compléments: une chronologie de la nouvelle en Europe aux xiv-xvi siècles (Annexe 1), des tableaux des emprunts permettant de suivre la circulation des sujets d'un recueil à l'autre (Annexe 2), des tableaux sur la structure des recueils, nécessairement adaptés à chaque texte (Annexe 3). La bibliographie est précieuse, non seulement par la quantité impressionnante des entrées (2169 notices!), mais également par son organisation interne (on se rapportera au sommaire pp. 875-878) et par sa mise à jour.

8 On me pardonnera, en conclusion, une remarque stylistique, qui n'enlève rien à la valeur d'ensemble de l'ouvrage: la profusion des images, des métaphores, des jeux de mots et des figures (la paronymie par-dessus tout) fatigue le lecteur, continuellement sollicité à 'traduire' les feux d'artifice stylistiques en des expressions sans aucun doute plus banales mais certainement plus claires, et nuit parfois à la clarté de l'exposé; mais peut-on honnêtement formuler un tel reproche à l'égard d'une savante qui subit très visiblement le charme de la prose $\mathrm{du} \mathrm{xv}^{\mathrm{e}}$ siècle? 